Article

\title{
Impact of Vitamin D Deficiency on COVID-19-A Prospective Analysis from the CovILD Registry
}

\author{
Alex Pizzini ${ }^{1, \dagger}{ }^{\dagger}$, Magdalena Aichner ${ }^{1,+}$, Sabina Sahanic ${ }^{1}$, Anna Böhm ${ }^{1}$, Alexander Egger ${ }^{2}$, \\ Gregor Hoermann ${ }^{2,3,4}$, Katharina Kurz ${ }^{1}$, Gerlig Widmann ${ }^{5}$, Rosa Bellmann-Weiler ${ }^{1}$, \\ Günter Weiss ${ }^{1,6}{ }^{\mathbb{D}}$, Ivan Tancevski ${ }^{1}$, Thomas Sonnweber ${ }^{1, *,+}$ and Judith Löffler-Ragg ${ }^{1}$ \\ 1 Department of Internal Medicine II, Infectious Diseases, Pneumology, Rheumatology, \\ Medical University of Innsbruck, 6020 Innsbruck, Austria; alex.pizzini@i-med.ac.at (A.P.); \\ magdalena.aichner@student.i-med.ac.at (M.A.); sabina.sahanic@i-med.ac.at (S.S.); \\ anna-k.boehm@student.i-med.ac.at (A.B.); katharina.kurz@i-med.ac.at (K.K.); \\ rosa.bellmann-weiler@i-med.ac.at (R.B.-W.); guenter.weiss@i-med.ac.at (G.W.); \\ ivan.tancevski@i-med.ac.at (I.T.); Judith.loeffler@i-med.ac.at (J.L.-R.) \\ 2 Central Institute of Medical and Chemical Laboratory Diagnostics, Medical University of Innsbruck, \\ 6020 Innsbruck, Austria; alexander.egger@tirol-kliniken.at (A.E.); gregor.hoermann@tirol-kliniken.at (G.H.) \\ 3 Department of Laboratory Medicine, Medical University of Vienna, 1090 Vienna, Austria \\ 4 MLL Munich Leukemia Laboratory, Klinikum Großhadern, 81377 Munich, Germany \\ 5 Department of Radiology, Medical University of Innsbruck, 6020 Innsbruck, Austria; \\ gerlig.widmann@tirol-kliniken.at \\ 6 Christian Doppler Laboratory for Iron Metabolism and Anemia Research, Medical University of Innsbruck, \\ 6020 Innsbruck, Austria \\ * Correspondence: thomas.sonnweber@i-med.ac.at; Tel.: +43-(0)-512-504-82586 \\ + A.P. and M.A. contributed equally to this manuscript.
}

Received: 10 August 2020; Accepted: 9 September 2020; Published: 11 September 2020

check for updates

\begin{abstract}
The novel Coronavirus disease 2019 (COVID-19) caused by severe acute respiratory syndrome coronavirus type 2 (SARS-CoV-2) is a global health concern. Vitamin D (VITD) deficiency has been suggested to alter SARS-CoV-2 susceptibility and the course of disease. Thus, we aimed to investigate associations of VITD status to disease presentation within the CovILD registry. This prospective, multicenter, observational study on long-term sequelae includes patients with COVID-19 after hospitalization or outpatients with persistent symptoms. Eight weeks after PCR confirmed diagnosis, a detailed questionnaire, a clinical examination, and laboratory testing, including VITD status, were evaluated. Furthermore, available laboratory specimens close to hospital admission were used to retrospectively analyze 25-hydroxyvitamin D levels at disease onset. A total of 109 patients were included in the analysis (60\% males, $40 \%$ females), aged $58 \pm 14$ years. Eight weeks after the onset of COVID-19, a high proportion of patients presented with impaired VITD metabolism and elevated parathyroid hormone (PTH) levels. PTH concentrations were increased in patients who needed intensive care unit (ICU) treatment, while VITD levels were not significantly different between disease severity groups. Low VITD levels at disease onset or at eight-week follow-up were not related to persistent symptom burden, lung function impairment, ongoing inflammation, or more severe CT abnormalities. VITD deficiency is frequent among COVID-19 patients but not associated with disease outcomes. However, individuals with severe disease display a disturbed parathyroid-vitamin-D axis within their recovery phase. The proposed significance of VITD supplementation in the clinical management of COVID-19 remains elusive.
\end{abstract}

Keywords: vitamin D; VITD; COVID-19; SARS-CoV-2; PTH; parathyroid hormone 


\section{Introduction}

The novel Coronavirus disease 2019 (COVID-19) caused by severe acute respiratory syndrome coronavirus type 2 (SARS-CoV-2) is a global health concern leading to a substantial need for patient hospitalization, treatment at intensive care units (ICUs), and invasive ventilation [1-3]. There is a risk of morbidity and mortality from COVID-19 as a consequence of severe pulmonary involvement and multi-organ failure varies across the general population. Several risk factors impacting the clinical course of COVID-19 have been described in the literature [4-8], and in most of these conditions, vitamin D (VITD) deficiency occurs frequently, especially in advanced age [9].

VITD, traditionally known as a crucial regulator of bone metabolism, is obtained either from nutritional sources or endogenous production. The endogenous generation of 25 -hydroxyvitamin $\mathrm{D}$ $(25(\mathrm{OH}) \mathrm{D})$, the major circulating form of VITD, includes various enzymatic steps. One crucial step in $25(\mathrm{OH}) \mathrm{D}$ production, the transformation of 7-dehydrocholesterol to previtamin D3, takes place in the skin and depends on the action of UV light intensity and duration [10]. Thus, VITD deficiency is related to seasonal changes, being most prevalent during winter season, and is very common in general, especially in developed countries. Ultimately, the fully active hormone is generated by hydroxylation at position 1 in the kidney to form 1,25-dihydroxyvitamin $\mathrm{D}(1,25(\mathrm{OH}) \mathrm{D})$ [11]. This final step is catalyzed by CYP27B1, an enzyme also found in many extrarenal tissues, which are able to produce $1,25(\mathrm{OH}) \mathrm{D}$ in a para- or autocrine manner [12].

As suggested by the Task Force of the Endocrine Society, VITD deficiency is determined by measurement of serum $25(\mathrm{OH}) \mathrm{D}$, which represents the most robust indicator to monitor the VITD status [13]. Accordingly, VITD deficiency is defined by a serum $25(\mathrm{OH}) \mathrm{D}$ concentration below $30 \mathrm{nmol} / \mathrm{L}$, whereas serum 25(OH)D concentration below $50 \mathrm{nmol} / \mathrm{L}$ indicates insufficient VITD supply. Based on these definitions, up to 40 percent of European individuals suffer from VITD deficiency [14,15].

Besides, various non-skeletal functions have been associated with VITD metabolism [16]. For instance, VITD interacts with the RAAS system, thus altering vascular wall tension and blood pressure, and may prevent the establishment and progression of atherosclerosis [17,18]. Additionally, VITD is related to immune surveillance and contributes to defense against bacterial and viral infections $[19,20]$. In this context, previous studies revealed a higher susceptibility to seasonal influenza and respiratory syncytial virus infections in VITD-deficient subjects [20,21]. VITD deficiency was further related to cases of severe pneumonia and the development of acute lung injury [22]. Since these conditions may occur as clinical features of a SARS-CoV-2 infection, we scrutinized the impact of VITD metabolism on the clinical course of COVID-19. The potential benefits of normal-ranged VITD levels in COVID-19 have previously been suggested; however, published studies on the impact of VITD status on the course of COVID-19 are lacking [20]. Moreover, public interest in VITD seems to be even more significant since the SARS-CoV-2 outbreak. Google trends revealed a significant boost in the frequency of "Vitamin D" being looked up during the SARS-CoV-2 pandemic [23]. Thus, we aimed to analyze VITD status and its associations with clinical presentation and course of disease in COVID-19.

\section{Materials and Methods}

Herein, we report results of the ongoing prospective multicenter observational CovILD study (ClinicalTrials.gov number, NCT04416100), aiming to evaluate the persistent cardio-pulmonary damage of COVID-19 patients. This prospective, multicentre, observational study includes patients with a confirmed diagnosis of COVID-19, based on typical clinical presentation and a positive SARS-CoV-2 real-time PCR test. The target population included hospitalized patients as well as outpatients with persistent symptoms. Inclusion criteria were female and male patients $\geq 18$ years with a confirmed infection with SARS-CoV-2 according to the definition of the Austrian Federal Ministry of Social Affairs, Health, Care, and Consumer Protection, and signed and dated declaration of consent by the patient according to ICH-GCP Guidelines [24]. Exclusion criteria were pregnancy, dementia, or declaration of consent by the patient according to ICH-GCP Guidelines not signed. 
Enrollment of patients with confirmed SARS-CoV-2 infection began on 29 April 2020. The trial site was located in Innsbruck, with two additional participating centers in Zams and Münster, all care centers in Tyrol, the first major COVID-19 hotspot in Austria. Due to the sudden rise in infections, patients had to be treated in wards from different medical disciplines. In total, the study cohort included 22 outward and 87 hospitalized patients, of whom 18 patients needed treatment at the ICU. Eight weeks after the confirmed diagnosis, a detailed questionnaire, clinical examination; and lung function testing including spirometry, body plethysmography, exhaled nitric oxide (FeNO), diffusion capacity for carbon monoxide adjusted for haemoglobin (DLCOc), capillary blood gas analysis, trans-thoracic echocardiography, standard laboratory examinations, a low-dose computed tomography (CT) scan of the chest, and laboratory testing, were evaluated. Laboratory parameters relevant for this analysis included $25(\mathrm{OH}) \mathrm{D}(\mathrm{nmol} / \mathrm{L})$, parathyroid hormone $(\mathrm{PTH})(\mathrm{ng} / \mathrm{L})$, calcium (total and ionized, $\mathrm{nmol} / \mathrm{L})$, phosphate $(\mathrm{mmol} / \mathrm{L})$, creatinine $(\mathrm{mg} / \mathrm{dL})$, urea $(\mathrm{mg} / \mathrm{dL})$, C-reactive-protein (CRP), interleukin-6 (IL-6), serum ferritin, and D-dimer. Additionally, specimens of 37 patients acquired during the first days of hospital admission were used to retrospectively determine $25(\mathrm{OH})$ levels at disease onset. According to current guidelines, VITD deficiency was defined as 25(OH)D levels below $30 \mathrm{nmol} / \mathrm{L}$. Serum concentrations of 25(OH)D between 30 and $50 \mathrm{nmol} / \mathrm{L}$ were categorized as insufficient VITD supply, whereas 25(OH)D above $100 \mathrm{nmol} / \mathrm{L}$ were considered normal [12]. Disease severity was categorized as mild for patients in outward treatment; moderate for patients in inward treatment; and severe for patients requiring oxygen supply, respiratory support, or intensive care treatment.

CT images were evaluated for the presence of ground-glass opacities (GGO), consolidations, bronchiectasis, and reticulations, as defined by the glossary of terms of the Fleischner society, and the intensity of the findings was graded according to their distribution (unilateral/bilateral, involved lobes) by assigning a score ranging from $0-5$ per lobe [25].

Mean comparison of normally distributed numeric data was performed using Student's $t$-test. If Gaussian distribution was not given, the Mann-Whitney-U-test and Kruskal-Wallis-test were applied. Spearman rank correlation coefficient was used for the analysis of monotonic associations in non-normally distributed data. If Gaussian distribution was given, Pearson correlation coefficient was calculated to assess the degree of linear associations. All tests were calculated two-tailed, and a $p$-value of 0.05 indicated statistical significance. Statistical analyses were performed with SPSS 24.0 statistical package (IBM Corp., Armonk, NY, USA).

All procedures performed in the present study involving human participants were in accordance with the ethical standards of the Institutional and/or National Research Committee and with the 1964 Helsinki declaration and its later amendments, and were performed after approval of the ethics committee of the Medical University of Innsbruck (EK Nr: 1103/2020).

\section{Results}

The study cohort consisted of predominantly male individuals (60\%), aged $58 \pm 14$ years. Sixty-five percent of COVID-19 patients were overweight or obese, and most individuals had pre-existing comorbidities, with cardiovascular and endocrine diseases being the most frequent ones.

During hospitalization, most patients needed oxygen supply (53\%), and 21 percent were admitted to the ICU due to the necessity of non-invasive or invasive mechanical ventilation as determined by the treating physicians. Detailed demographics and clinical characteristics of the study cohort are shown in Table 1.

Eight weeks after onset of COVID-19, mean 25(OH)D concentrations were $54 \pm 25 \mathrm{nmol} / \mathrm{L}$, demonstrating a high proportion of patients with impaired VITD metabolism, and highlighting male patients, who displayed significantly lower $25(\mathrm{OH}) \mathrm{D}$ levels $(50 \mathrm{nmol} / \mathrm{L} \pm 23 \mathrm{vs} .61 \pm 25 \mathrm{nmol} / \mathrm{L}, p=0.01)$ than females. Overall, $12 \%$ of patients presented with VITD deficiency and $41 \%$ with VITD insufficiency. Accordingly, PTH was significantly increased $(>65 \mathrm{ng} / \mathrm{L})$ in 13 percent of patients. From 37 patients, $25(\mathrm{OH}) \mathrm{D}$ levels could be retrospectively assessed from samples stored at the moment of COIVD-19 
diagnosis. The mean 25(OH)D concentration was $49 \pm 36 \mathrm{nmol} / \mathrm{L}$. During hospitalization, 38\% of patients had 25(OH)D deficiency and 27\% 25(OH) insufficiency (Figure 1).

Table 1. Demographics and clinical characteristics of patients with COVID-19.

\begin{tabular}{cc}
\hline Characteristics & $(\mathbf{N}=\mathbf{1 0 9})$ \\
\hline Median age (SD)-yr & $58 \pm 14$ \\
Female sex-no. (\%) & $44(40)$ \\
Mean body mass index-kg/m (SD) $^{*}$ & $27 \pm 14$ \\
\hline Comorbidities No. (\%) & \\
\hline None & $21(19)$ \\
Cardiovascular disease & $44(40)$ \\
Hypertension & $32(29)$ \\
Pulmonary disease & $21(19)$ \\
Endocrine disease & $49(45)$ \\
Hypercholesterolemia & $24(22)$ \\
Diabetes, type 2 & $20(18)$ \\
Chronic kidney disease & $7(6)$ \\
Chronic liver disease & $6(6)$ \\
Malignancy & $16(15)$ \\
Immunodeficiency & $7(6)$ \\
\hline Treatment & \\
\hline Oxygen supply-no. (\%) & $53(49)$ \\
Non-invasive ventilation-no. (\%) & $2(2)$ \\
Invasive ventilation-no. (\%) & $16(15)$ \\
Vitamin-D supplementation & $10(9)$ \\
\hline
\end{tabular}

* The body-mass index (BMI) is the weight kilograms divided by the square of the height in meters.

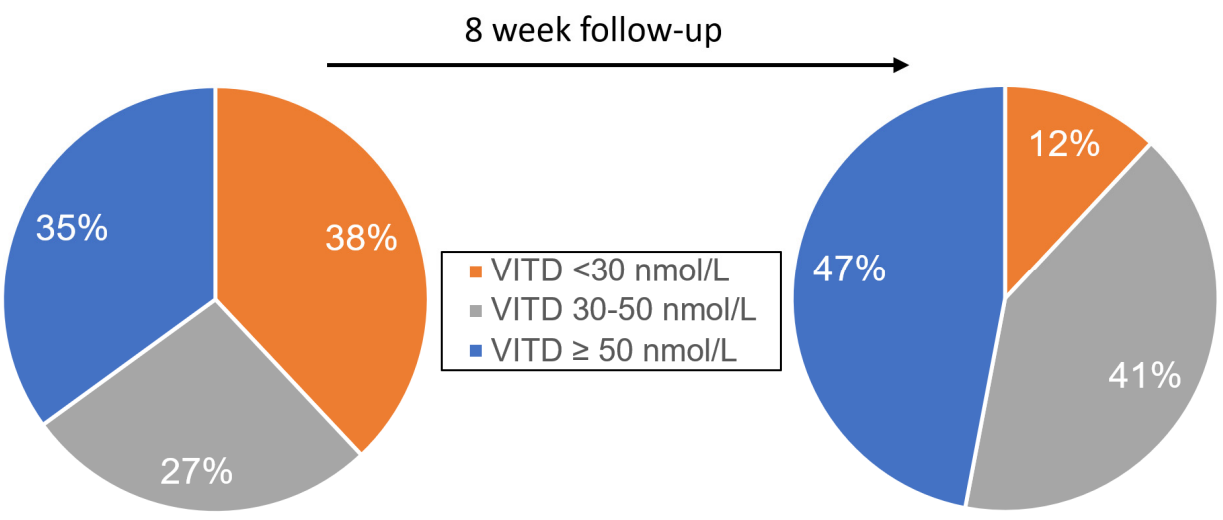

Figure 1. Vitamin D during hospitalization and after 8-week follow-up. Data is presented as percentage and categorized into Vitamin D (VITD) concentrations $<30 \mathrm{nmol} / \mathrm{L}, 30-50 \mathrm{nmol} / \mathrm{L}$, and $>50 \mathrm{nmol} / \mathrm{L}$. On the left, data during hospitalization is compared to data from 8-week follow-up on the right.

The 25(OH)D concentrations at diagnosis highly correlated with those at the first follow-up visit $(\mathrm{r}=0.805, p<0.01)$, and significant rise in $25(\mathrm{OH}) \mathrm{D}$ concentrations was noted during the observation period $(p=0.01)$.

PTH concentrations were more significantly elevated in subjects who needed ICU or prolonged oxygen treatment $(p<0.01)$ than in mild cases. Similar differences were noted when comparing 25(OH)D levels among mild, moderate, and severe groups, although they did not reach statistical significance. Still, when comparing $25(\mathrm{OH}) \mathrm{D}$ levels of the severe subgroup to pooled data of mild and moderate cases, they were significantly lower $(58 \pm 25$ vs. $50 \pm 24 \mathrm{nmol} / \mathrm{L}, p<0.05)$ at follow-up. In contrast, $25(\mathrm{OH}) \mathrm{D}$ concentrations at disease onset did not differ significantly between these groups. $(p=0.20) .(p=0.12$; Figure 2, Table 2$)$. 
Table 2. Laboratory parameters in COVID-19 patients 8 weeks after disease onset.

\begin{tabular}{|c|c|c|c|c|c|}
\hline & $\begin{array}{c}\text { Total } \\
(N=109)\end{array}$ & $\begin{array}{c}\text { Mild } \\
(N=22)\end{array}$ & $\begin{array}{c}\text { Moderate } \\
(N=34)\end{array}$ & $\begin{array}{l}\text { Severe * } \\
(N=53)\end{array}$ & $p$-Value \\
\hline Median age (SD)-yr & $58 \pm 14$ & $46 \pm 16$ & $60 \pm 13$ & $61 \pm 12$ & 0.001 \\
\hline Female sex-no. (\%) & $44(40)$ & $14(64)$ & $20(60)$ & $10(19)$ & 0.001 \\
\hline Mean BMI-kg/m² (SD) † & $27 \pm 14$ & $26 \pm 5$ & $26 \pm 4$ & $28 \pm 5$ & 0.287 \\
\hline Days of hospitalization & $9 \pm 10$ & $0(0)$ & $5 \pm 3$ & $15 \pm 10$ & $<0.001$ \\
\hline $25(\mathrm{OH}) \mathrm{D} \mathrm{nmol} \cdot \mathrm{L}^{-1}$ & $54 \pm 25$ & $64 \pm 31$ & $54 \pm 19$ & $50 \pm 24$ & 0.116 \\
\hline PTH-ng. $\mathrm{L}^{-1}$ & $45 \pm 18$ & $35 \pm 13$ & $42 \pm 14$ & $50 \pm 20$ & 0.001 \\
\hline \multicolumn{6}{|l|}{ Calcium-mmol $\cdot \mathrm{L}^{-1}$} \\
\hline total & $2.37 \pm 0.09$ & $2.37 \pm 0.09$ & $2.36 \pm 0.09$ & $2.39 \pm 0.08$ & 0.183 \\
\hline ionized & $1.22 \pm 0.04$ & $1.24 \pm 0.03$ & $1.22 \pm 0.04$ & $1.22 \pm 0.04$ & 0.310 \\
\hline Phosphate-mmol $\cdot \mathrm{L}^{-1}$ & $1.01 \pm 0.17$ & $1.02 \pm 0.14$ & $1.04 \pm 0.16$ & $0.99 \pm 0.19$ & 0.473 \\
\hline Creatinine $-\mathrm{mg} \cdot \mathrm{dL}^{-1}$ & $0.87 \pm 0.23$ & $0.82 \pm 0.15$ & $0.80 \pm 0.17$ & $0.93 \pm 0.27$ & 0.017 \\
\hline Urea-mg.dL $\mathrm{L}^{-1}$ & $32 \pm 11$ & $28 \pm 7$ & $32 \pm 9$ & $33 \pm 13$ & 0.242 \\
\hline $\mathrm{CRP}-\mathrm{mg} \cdot \mathrm{dL}^{-1}$ & $0.29 \pm 0.44$ & $0.2 \pm 0.28$ & $0.2 \pm 0.21$ & $0.39 \pm 0.56$ & 0.067 \\
\hline IL-6-ng $\cdot \mathrm{L}^{-1}$ & $3.1 \pm 4.98$ & $1.45 \pm 2.06$ & $1.96 \pm 1.95$ & $4.43 \pm 6.6$ & 0.041 \\
\hline D-dimer-ug. $\mathrm{L}^{-1}$ & $807 \pm 1591$ & $607 \pm 797$ & $632 \pm 633$ & $1001 \pm 2160$ & 0.475 \\
\hline Ferritin-ug $\cdot \mathrm{L}^{-1}$ & $263 \pm 230$ & $139 \pm 118$ & $260 \pm 183$ & $317 \pm 271$ & 0.001 \\
\hline
\end{tabular}

+ BMI, body-mass index; the BMI is the weight kilograms divided by the square of the height in meters. * 35 patients received oxygen supply only, two patients were treated with non-invasive ventilation, and 16 with invasive ventilation. Disease severity was graded according to intensity of treatment: mild = ambulatory treatment; moderate $=$ hospital treatment; severe = inward treatment with respiratory (oxygen) supply or treatment at the intensive care unit (ICU) with non-invasive or invasive ventilation; $\mathrm{PTH}=$ parathyroid hormone; $\mathrm{CRP}=\mathrm{C}$-reactive protein; IL-6 = interleukin 6; data are depicted as mean \pm SD, p-values were calculated with Kruskal-Wallis Test.

A)

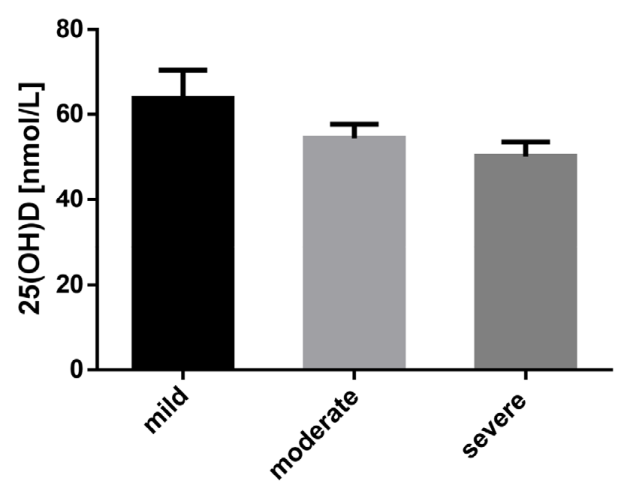

B)

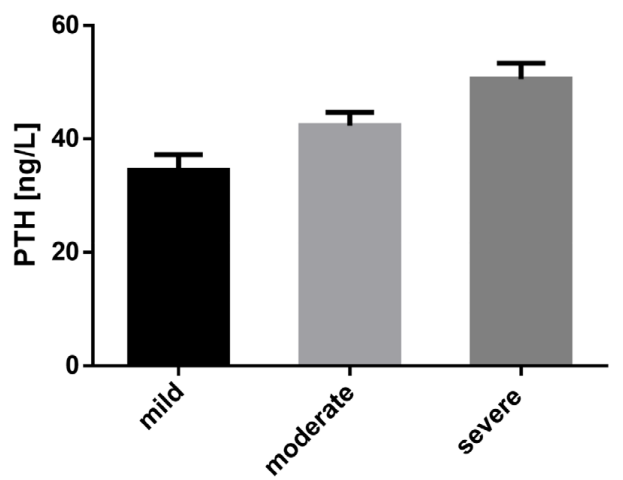

Figure 2. Vitamin D (A) and parathyroid hormone (PTH) (B) concentration according to disease severity at eight weeks follow-up: serum concentrations of $(\mathbf{A}) 25$-hydroxyvitamin $\mathrm{D}(25(\mathrm{OH}) \mathrm{D})$ and (B) PTH. Disease severity was graded according to intensity of treatment: mild = ambulatory treatment; moderate $=$ hospital treatment; severe=inward treatment with respiratory (oxygen) supply or treatment at the ICU with non-invasive or invasive ventilation.

When we compared 25(OH)D levels in patients with or without $\mathrm{CT}$ abnormalities, no significant difference was apparent (55nmol/L vs. $54 \mathrm{nmol} / \mathrm{L}, p=0.83)$. Accordingly, 25(OH)D concentrations in patients with normal or impaired lung function did not show significant differences either at disease onset ( $48 \mathrm{vs.} 50 \mathrm{nmol} / \mathrm{L}, p=0.84$ ) or at follow-up ( $57 \mathrm{vs.} 50 \mathrm{nmol} / \mathrm{L}, p=0.15)$, and 25(OH)D levels at disease onset did not predict symptom burden at follow-up. The same was the case when analyzing PTH levels in regards to CT abnormalities ( $p=0.07)$, impaired lung function $(p=0.89)$, and persistent symptoms $(p=0.82)$. Correlation analysis between $25(\mathrm{OH}) \mathrm{D}$ levels at follow-up and CRP $(\mathrm{r}=-0.021$, $p=0.88)$, IL-6 $(\mathrm{r}=-0.003, p=0.98)$, serum ferritin $(\mathrm{r}=0.019, p=0.89)$, and $\mathrm{D}$-dimer $(\mathrm{r}=-0.155, p=0.26)$ revealed no significant associations. The same was true for $25(\mathrm{OH}) \mathrm{D}$ levels at disease onset and CRP 
$(\mathrm{r}=0.152, p=0.45), \mathrm{IL}-6(\mathrm{r}=0.050, p=0.80)$, and serum ferritin $(\mathrm{r}=0.070, p=0.73)$. In contrast, D-Dimer levels were moderately associated with 25(OH)D levels at disease onset $(r=0.437, p<0.05)$.

Ten $(9.2 \%)$ of patients received VITD supplementation during hospitalization, but the initiation of VITD supplementation was not related to disease severity.

\section{Discussion}

The herein-presented analysis of the CovILD study cohort shows that $25(\mathrm{OH}) \mathrm{D}$ deficiency is common among COVID-19 patients, whereas a causal implication of VitD metabolism on its disease course remains uncertain.

Evidence from a study by Hastie et al., who retrospectively analyzed the influence of vitamin D status on COVID-19 infection risk, supports the here presented results. Comparison of 25(OH)D levels of COVID-19 patients with UK Biobank data did not support a potential role for VITD metabolism for the susceptibility to COVID-19 infection nor for the differences between ethnic groups [26]. As pointed out in the response by Roy et al., the study focused on the association of VITD to the risk of incidence of COVID-19 rather than the risk of severity [27]. Contrasting results are reported from an Israeli population-based study, which, similar to this study, identified a high frequency of $25(\mathrm{OH}) \mathrm{D}$ deficiency in COVID-19 patients. Multivariate analysis, after controlling for demographic characteristics and medical conditions, confirmed an independent and significant association between a low $25(\mathrm{OH}) \mathrm{D}$ level and an increased likelihood of COVID-19 infection [28].

This prospective observational cohort study reveals first evidence of a disturbed PTH-VITD axis in patients with a more severe course of COVID-19. Low levels of $25(\mathrm{OH}) \mathrm{D}$, however, did not predict the severity of the disease and did not associate with persistent symptoms, CT-abnormalities, or impaired pulmonary function testing, either at the moment of COVID-19 diagnosis or at the 8-week follow-up. Nevertheless, the high proportion of patients with elevated PTH concentrations, especially in severe COVID-19 cases during the recovery phase, is remarkable. This might simply reflect the result of less sunlight exposure as a consequence of prolonged quarantine periods and hospitalization, leading to secondary hyperparathyroidism, or represent a residual dysregulation after the infectious disease [29]. Patients' reduced mobility due to persistent COVID-19 related symptoms such as fatigue and dyspnoea may also contribute to the described alterations. The recently observed peaks in new SARS-CoV-2 infections in regions with high sunlight exposure and consequently expected abundant endogenous VITD synthesis, like Florida and California, further questions the significance of VITD on COVID-19 susceptibility and disease course, contrasting with the so-called latitude hypothesis [30].

Results from a small Italian study including 42 COVID-19 patients with respiratory failure treated at the ICU identified $81 \%$ of the patients having $25(\mathrm{OH}) \mathrm{D}$ deficiency. Severe deficiency, defined as 25(OH)D below $10 \mathrm{ng} / \mathrm{mL}$, was identified in 10 patients and associated with a significant elevation in mortality, despite being by far the oldest study-subgroup with comorbidities in every patient. A direct comparison with our study is inappropriate as study-design and outcome-measurements distinctly differ; however, a proper validation in a larger cohort of ICU patients is warranted in order to draw robust conclusions related to the prognostic impact of VITD deficiency in critically ill COVID-19 patients.

The absolute increase in serum 25(OH)D levels from the time of COVID-19 diagnosis to follow-up is subject for discussion. As 25(OH)D levels were not routinely analyzed in COVID-19 patients during hospitalization, we used laboratory specimens close to hospital admission to retrospectively determine $25(\mathrm{OH}) \mathrm{D}$ levels. Still, laboratory specimens were only available from a subgroup of the cohort $(n=37)$, thus results of this analysis have to be interpreted with caution. Additionally, patients with a severe lack of 25(OH)D received VITD supplementation as part of the clinical management.

The immunomodulatory effects of VITD described in the context of pneumonia, acute lung injury, and systemic inflammatory response syndrome would sustain a potential link to COVID-19 and imply a possibility for intervention [31]. The known interaction of VITD with the renin-angiotensin system, including ACE2, whose receptor SARS-Cov-2 binds to, further recommends it as a potential therapeutic option [32]. Accordingly, in a rat model, VITD has already been shown to alleviate acute lung injury by 
modulating the renin-angiotensin system [33]. However, previous reports also question the causal implication of VITD deficiency, especially low 25(OH)D levels, in extra-skeletal health [29,34] by discussing the potential ability of infections and chronic inflammatory processes in reducing serum $25(\mathrm{OH})$ D levels. Such causal association would also explain why VITD insufficiency/deficiency is reported in a wide range of disorders, although results of randomized controlled trials using vitamin D supplementation in preventing or ameliorating extra-skeletal diseases are mostly inconsistent or even disappointing [34,35].

Although the abovementioned studies justify further analyses, including 25(OH)D interventional studies, in COVID-19, our results do not support VITD deficiency as a strong indicator of severe disease course. However, as the CovILD study was not designed to evaluate the effects of $25(\mathrm{OH}) \mathrm{D}$ in COVID-19, we have to acknowledge some limitations. First, the CovILD trial was designed as an observational study to detect the rate of persistent lung injury after a severe course of COVID-19, therefore the here presented results must be interpreted carefully in the context of VITD deficiency, especially because predominantly patients with severe disease course were included. Second, 25(OH)D levels were prospectively assessed eight weeks after the positive SARS-Cov-2 PCR test, while only a third of the study population $25(\mathrm{OH}) \mathrm{D}$ levels at disease onset were retrospectively available. Thus, further prospective clinical studies are needed to clarify the significance of VITD in the clinical management of COVID-19 patients.

\section{Conclusions}

VITD deficiency is frequently found in patients with severe COVID-19, but 25(OH)D concentrations do not associate with persistent inflammation, impairment in pulmonary function tests, pathological findings in CT-scans, or the persistence of symptoms. However, individuals with severe disease display a disturbed parathyroid-vitamin-D axis within their recovery phase, most likely due to prolonged hospitalization, although the question about causality or consequence cannot be answered through our data, and more evidence from interventional RCTs is warranted to properly understand the role of VITD in COVID-19. Conclusively, the proposed significance of VITD in the clinical management of COVID-19 remains elusive.

Author Contributions: A.P.: wrote the manuscript, contributed to the conception and design of the study, the acquisition of data, and the analysis and interpretation; M.A.: contributed to the conception and design of the study, the acquisition of data, and the analysis and interpretation and co-wrote the paper; S.S.: contributed to the conception and design of the study and the acquisition of data; A.B.: contributed to the acquisition of data; A.E.: contributed to the acquisition of data and data analysis; G.H.: contributed to the acquisition of data and data analysis; K.K.: contributed to the acquisition of data; G.W. (Gerlig Widmann): data analysis and interpretation; R.B.-W.: contributed to the acquisition of data; G.W. (Günter Weiss): conception and design of the study, data analysis, and interpretation; I.T.: conception and design of the study; T.S.: study design, data analysis and interpretation, and co-wrote the paper, corresponding author; J.L.-R.: conception and design of the study, data analysis, and interpretation. All authors have read and agreed to the published version of the manuscript.

Funding: I.T. was awarded an Investigator Initiated Study (IIS) grant by by Boehringer Ingelheim (IIS 1199-0424).

Acknowledgments: We acknowledge Doris Hüttenberger and Graziella Plank for supporting this project. Support by the "Verein zur Förderung von Forschung und Weiterbildung in Infektiologie und Immunologie an der Medizinischen Universität Innsbruck“ (ZVR-Zahl 474802789, GZ: LVR 2233) is gratefully acknowledged.

Conflicts of Interest: The authors declare no conflict of interest.

\section{References}

1. WHO. Coronavirus Disease 2019. 2020. Available online: https://www.who.int/emergencies/diseases/novelcoronavirus-2019/events-as-they-happen (accessed on 23 June 2020).

2. CfDCaP. Coronavirus (COVID-19). 2020. Available online: https://www.cdc.gov/coronavirus/2019-ncov/ index.html (accessed on 23 June 2020).

3. ECfDPaC. COVID-19 Pandemic. 2020. Available online: https://www.ecdc.europa.eu/en/covid-19-pandemic (accessed on 23 June 2020). 
4. Cummings, M.J.; Baldwin, M.R.; Abrams, D.; Jacobson, S.D.; Meyer, B.J.; Balough, E.M.; Aaron, J.G.; Claassen, J.; Rabbani, L.E.; Hastie, J.; et al. Epidemiology, clinical course, and outcomes of critically ill adults with COVID-19 in New York City: A prospective cohort study. Lancet 2020, 395, 1763-1770. [CrossRef]

5. Du, R.H.; Liang, L.R.; Yang, C.Q.; Wang, W.; Cao, T.Z.; Li, M.; Guo, G.Y.; Du, J.; Zheng, C.L.; Zhu, Q.; et al. Predictors of mortality for patients with COVID-19 pneumonia caused by SARS-CoV-2: A prospective cohort study. Eur. Respir. J. 2020, 55. [CrossRef] [PubMed]

6. Ellinghaus, D.; Degenhardt, F.; Bujanda, L.; Buti, M.; Albillos, A.; Invernizzi, P.; Fernández, J.; Prati, D.; Baselli, G.; Asselta, R.; et al. Genomewide Association Study of Severe Covid-19 with Respiratory Failure. N. Engl. J. Med. 2020. [CrossRef]

7. Zhou, F.; Yu, T.; Du, R.; Fan, G.; Liu, Y.; Liu, Z.; Xiang, J.; Wang, Y.; Song, B.; Gu, X.; et al. Clinical course and risk factors for mortality of adult inpatients with COVID-19 in Wuhan, China: A retrospective cohort study. Lancet 2020, 395, 1054-1062. [CrossRef]

8. Williamson, E.J.; Walker, A.J.; Bhaskaran, K.; Bacon, S.; Bates, C.; Morton, C.E.; Curtis, H.J.; Mehrkar, A.; Evans, D.; Inglesby, P.; et al. OpenSAFELY: Factors associated with COVID-19 death in 17 million patients. Nature 2020, 584, 430-436. [CrossRef]

9. Marino, R.; Misra, M. Extra-Skeletal Effects of Vitamin D. Nutrients 2019, 11, 1460. [CrossRef]

10. Bikle, D.D. Vitamin D metabolism, mechanism of action, and clinical applications. Chem. Biol. 2014, 21, 319-329. [CrossRef]

11. Pludowski, P.; Holick, M.F.; Grant, W.B.; Konstantynowicz, J.; Mascarenhas, M.R.; Haq, A.; Povoroznyuk, V.; Balatska, N.; Barbosa, A.P.; Karonova, T.; et al. Vitamin D supplementation guidelines. J. Steroid Biochem. Mol. Biol. 2018, 175, 125-135. [CrossRef]

12. Giustina, A.; Adler, R.A.; Binkley, N.; Bouillon, R.; Ebeling, P.R.; Lazaretti-Castro, M.; Marcocci, C.; Rizzoli, R.; Sempos, C.T.; Bilezikian, J.P. Controversies in Vitamin D: Summary Statement From an International Conference. J. Clin. Endocrinol. Metab. 2019, 104, 234-240. [CrossRef]

13. Holick, M.F.; Binkley, N.C.; Bischoff-Ferrari, H.A.; Gordon, C.M.; Hanley, D.A.; Heaney, R.P.; Murad, M.H.; Weaver, C.M. Evaluation, treatment, and prevention of vitamin D deficiency: An Endocrine Society clinical practice guideline. J. Clin. Endocrinol. Metab. 2011, 96, 1911-1930. [CrossRef]

14. Cashman, K.D.; Dowling, K.G.; Škrabáková, Z.; Gonzalez-Gross, M.; Valtueña, J.; De Henauw, S.; Moreno, L.; Damsgaard, C.T.; Michaelsen, K.F.; Mølgaard, C.; et al. Vitamin D deficiency in Europe: Pandemic? Am. J. Clin. Nutr. 2016, 103, 1033-1044. [CrossRef] [PubMed]

15. Liu, X.; Baylin, A.; Levy, P.D. Vitamin D deficiency and insufficiency among US adults: Prevalence, predictors and clinical implications. Br. J. Nutr. 2018, 119, 928-936. [CrossRef] [PubMed]

16. Wang, T.J. Vitamin D and Cardiovascular Disease. Annu. Rev. Med. 2016, 67, 261-272. [CrossRef] [PubMed]

17. Muscogiuri, G.; Annweiler, C.; Duval, G.; Karras, S.; Tirabassi, G.; Salvio, G.; Balercia, G.; Kimball, S.; Kotsa, K.; Mascitelli, L.; et al. Vitamin D and cardiovascular disease: From atherosclerosis to myocardial infarction and stroke. Int. J. Cardiol. 2017, 230, 577-584. [CrossRef]

18. Legarth, C.; Grimm, D.; Wehland, M.; Bauer, J.; Krüger, M. The Impact of Vitamin D in the Treatment of Essential Hypertension. Int. J. Mol. Sci. 2018, 19, 455. [CrossRef]

19. Bikle, D.D. Vitamin D and immune function: Understanding common pathways. Curr. Osteoporos. Rep. 2009, 7, 58-63. [CrossRef]

20. Grant, W.B.; Lahore, H.; McDonnell, S.L.; Baggerly, C.A.; French, C.B.; Aliano, J.L.; Bhattoa, H.P. Evidence that Vitamin D Supplementation Could Reduce Risk of Influenza and COVID-19 Infections and Deaths. Nutrients 2020, 12, 988. [CrossRef]

21. Gruber-Bzura, B.M. Vitamin D and Influenza-Prevention or Therapy? Int. J. Mol. Sci. 2018, 19, 2419. [CrossRef]

22. Parekh, D.; Thickett, D.R.; Turner, A.M. Vitamin D deficiency and acute lung injury. Inflamm. Allergy Drug Targets 2013, 12, 253-261. [CrossRef]

23. Google Trends. Explore. Available online: https://rends.google.com/trends/explore?date=today $\% 205-\mathrm{y} \& \mathrm{q}=$ $\% 2 F m \% 2 F 0 f 4 j p$ (accessed on 30 July 2020).

24. European Medicine Agency. ICH E6(R2) Good Clinical Practice (GCP) Guideline. Available online: https://www.ema.europa.eu/en/ich-e6-r2-good-clinical-practice (accessed on 30 July 2020).

25. Hansell, D.M.; Bankier, A.A.; MacMahon, H.; McLoud, T.C.; Muller, N.L.; Remy, J. Fleischner Society: Glossary of terms for thoracic imaging. Radiology 2008, 246, 697-722. [CrossRef] 
26. Hastie, C.E.; Mackay, D.F.; Ho, F.; Celis-Morales, C.A.; Katikireddi, S.V.; Niedzwiedz, C.L.; Jani, B.D.; Welsh, P.; Mair, F.S.; Gray, S.R.; et al. Vitamin D concentrations and COVID-19 infection in UK Biobank. Diabetes Metab. Syndr. 2020, 14, 561-565. [CrossRef]

27. Roy, A.S.; Matson, M.; Herlekar, R. Response to 'Vitamin D concentrations and COVID-19 infection in UK Biobank'. Diabetes Metab. Syndr. 2020, 14, 777. [CrossRef] [PubMed]

28. Merzon, E.; Tworowski, D.; Gorohovski, A.; Vinker, S.; Golan Cohen, A.; Green, I.; Frenkel-Morgenstern, M. Low plasma $25(\mathrm{OH})$ vitamin D level is associated with increased risk of COVID-19 infection: An Israeli population-based study. FEBS J. 2020, 287, 3693-3702. [CrossRef]

29. Mangin, M.; Sinha, R.; Fincher, K. Inflammation and vitamin D: The infection connection. Inflamm. Res. 2014, 63, 803-819. [CrossRef] [PubMed]

30. Hedlund, R.; Diamond, T.K.; Uversky, V.N. The latitude hypothesis, vitamin D, and SARS-Co-V2. J. Biomol. Struct. Dyn. 2020. [CrossRef] [PubMed]

31. Jakovac, H. COVID-19 and vitamin D-Is there a link and an opportunity for intervention? Am. J. Physiol. Endocrinol. Metab. 2020, 318, E589. [CrossRef]

32. Mahdavi, A.M. A brief review of interplay between vitamin D and angiotensin-converting enzyme 2: Implications for a potential treatment for COVID-19. Rev. Med. Virol. 2020. [CrossRef]

33. Xu, J.; Yang, J.; Chen, J.; Luo, Q.; Zhang, Q.; Zhang, H. Vitamin D alleviates lipopolysaccharide-induced acute lung injury via regulation of the renin-angiotensin system. Mol. Med. Rep. 2017, 16, 7432-7438. [CrossRef]

34. Al Nozha, O.M. Vitamin D and extra-skeletal health: Causality or consequence. Int. J. Health Sci. (Qassim) 2016, 10, 443-452. [CrossRef]

35. Theodoratou, E.; Tzoulaki, I.; Zgaga, L.; Ioannidis, J.P. Vitamin D and multiple health outcomes: Umbrella review of systematic reviews and meta-analyses of observational studies and randomised trials. BMJ 2014, 348, g2035. [CrossRef]

(C) 2020 by the authors. Licensee MDPI, Basel, Switzerland. This article is an open access article distributed under the terms and conditions of the Creative Commons Attribution (CC BY) license (http://creativecommons.org/licenses/by/4.0/). 